\title{
INVENTARIO DE LA FAUNA MARINA DE LA HISPANIOLA
}

\section{Alejandro Herrera-Moreno Liliana Betancourt-Fernández}

\section{RESUMEN}

El presente trabajo ofrece las primeras cifras representativas del conocimiento de la fauna marina de la Isla Hispaniola con un total de 2,359 especies pertenecientes a unos 44 grupos taxonómicos mayores. Para República Dominicana se hallaron 1,955 especies lo cual adiciona cerca de 900 nuevos registros al último inventario nacional de la biodiversidad marina. Para Haití, se hallaron 1,057 especies lo cual representa posiblemente el primer intento de resumir el conocimiento de su fauna marina. Las especies registradas son representativas de todos los ecosistemas y ambientes costeros y marinos -pelágicos y bentónicos- desde la costa hasta unos 3,000 m de profundidad. Comparativamente con otras islas de las Antillas Mayores, el conocimiento en número de especies de algunos grupos marinos puede considerarse avanzado en los peces o algunos grupos de invertebrados como corales escleractíneos, gorgonáceos, poliplacóforos o equinodermos; mientras que en otros grupos este conocimiento es pobre o incipiente. Los resultados del Proyecto HISPABIOTA constituyen un punto de partida para complementar y enriquecer los Reportes y las Estrategias Nacionales de la Biodiversidad de República Dominicana y Haití y constituyen una guía para nuevas investigaciones taxonómicas, ecológicas y zoogeográficas que contribuyan -sobre bases científicas- a la conservación, manejo y uso racional de la rica biodiversidad marina de la Isla Hispaniola. 


\section{INTRODUCCIÓN}

La Hispaniola -la segunda en extensión de las Antillas Mayores- es una isla alargada en sentido Este-Oeste, bañada por las aguas del Océano Atlántico al Norte y del Mar Caribe al Sur. Su parte oriental está ocupada por la República Dominicana, con una extensión de $48,730 \mathrm{~km}^{2}$ y la parte occidental por Haití con $27,750 \mathrm{~km}^{2}$. La extensión total de la Isla se estima en unos $76,480 \mathrm{~km}^{2}$, área que incluye varios cayos e islotes como las Islas Gonave, Tortue, Ile à Vache y Grand Cayemite, en la plataforma haitiana y Saona, Catalina y Beata en la dominicana. La plataforma marina que bordea este territorio es estrecha, con pendientes abruptas y abarca unos 13,310 $\mathrm{km}^{2}$, mientras que la línea litoral se extiende por unos 3,059 $\mathrm{km}$ de manglares de borde, lagunas costeras, playas arenosas, costas rocosas bajas o acantiladas, estuarios y bahías. Cerca de la costa se desarrollan fondos arenosos, fangosos o mixtos, frecuentemente con abundante macrovegetación de pastos marinos y/o macroalgas, como antesala a extensos arrecifes coralinos que se desarrollan hacia la región oceánica hasta profundidades aproximadas de $50 \mathrm{~m}$. En este mosaico de condiciones ambientales se desarrolla una exuberante y diversa fauna y flora marina, que ha acaparado la atención de los investigadores de todas partes del mundo durante varios siglos.

Sin embargo, los aportes al conocimiento de la biota marina de la Hispaniola se encuentran diseminados en las colecciones de museos de varios países, publicaciones internacionales referidas al ámbito caribeño y atlántico, publicaciones nacionales de escasa difusión o en reportes inéditos de proyectos, sin que hasta el momento se haya realizado un inventario global 
que permita evaluar el nivel de conocimiento en términos del número de especies de la biota marina insular. Este objetivo lo hace suyo el Proyecto HISPABIOTA del Programa EcoMar, Inc., cuyos resultados generales, correspondientes a la Fase I, se presentan en este trabajo.

\section{MATERIALES Y MÉTODOS}

Como parte de la Fase I del Proyecto HISPABIOTA: Búsqueda y compilación de antecedentes, se realizó una investigación bibliográfica que arrojó más de 400 estudios taxonómicos y/o ecológicos, con registros confiables de especies para las plataformas marinas y/o la región oceánica colindante de República Dominicana o Haití. Se consideraron además compilaciones de autores reconocidos, comunicaciones personales de fuentes acreditadas, resultados de las expediciones internacionales en aguas de la Hispaniola y nuestras propias colectas e identificaciones de campo. También se accedió a la información de unos 50 museos extranjeros mediante intercambio directo, literatura o vía Internet. A partir del análisis de esta información, se realizaron inventarios preliminares de especies ordenadas taxonómicamente para todos los grupos marinos posibles, siguiendo el orden de Ruppert y Barnes (1994) en lo general. En cada grupo se buscó la nomenclatura más actualizada y se consultaron autoridades reconocidas (por ejemplo Eschmeyer (1998) en peces) para la organización de las listas, como base para el conteo de especies. Solo las cifras de los conteos totales de especies/grupo se presentan en ese trabajo. Dos aspectos deben ser aclarados. Primero esta búsqueda ha sido realizada con los recursos a nuestro alcance, por lo que la misma es amplia y representativa, pero no exhaustiva. Segundo, los hallazgos de información no han sido equivalentes para todos los grupos, pues para algunos se hallaron numerosos trabajos e incluso monografías antiguas, mientras que en otros, 160 
se obtuvo información fragmentada que permitió obtener solamente una lista preliminar. Todas las posibles imprecisiones están siendo superadas en la Fase II de este Proyecto Actualización de inventarios de grupos particulares, que se enfoca en cada grupo en particular y que cuenta con la experiencia de nuestro trabajo previo sobre las macroalgas de la Hispaniola (ver Betancourt y Herrera, 2002).

\section{RESULTADOS Y DISCUSIÓN}

Preliminarmente nuestra búsqueda arroja que la fauna marina conocida de la Hispaniola podría estar representada por al menos 2,353 especies de invertebrados y peces, pertenecientes a unos 44 grupos taxonómicos mayores (Tabla 1), aunque estas cifras pueden variar con el examen de nueva información. Para República Dominicana registramos 1,955 especies y 1,057 para Haití Esta búsqueda incrementa en unas 900 especies y unos 14 nuevos taxa al último listado dominicano de CIBIMA (1994). Para Haití, este reporte constituye, hasta donde conocemos, el primer resumen del conocimiento de su biota marina. Las especies registradas son representativas de todos los ecosistemas y ambientes costeros y marinos, pelágicos y bentónicos, desde la costa hasta más de 3,000 $\mathrm{m}$ de profundidad.

El nivel de conocimiento general de algunos grupos de la fauna marina de la Hispaniola es comparable al que existe en otros países de las Antillas Mayores. Por ejemplo, nuestros registros de peces, con 901 especies, están al nivel del conocimiento de la ictiofauna cubana que reporta 906 especies (Claro 1994). No ocurre lo mismo con los invertebrados marinos a nivel global, pues comparativamente con la cifra de 3,948 especies conocidas que ofrece el Estudio de Biodiversidad de Cuba (MCTMA, 1998), nuestro conocimiento global a nivel de grandes grupos, con unas 1,461 especies conocidas, es aún pobre o incipiente. 
Tabla 1. Resumen preliminar del número de especies de varios grupos taxonómicos marinos de acuerdo a los resultados de la presente revisión. HA: Haití; RD: Dominicana; HS: Hispaniola. El orden general en invertebrados sigue a Ruppert y Barnes (1994) y en peces a Eschmeyer (1998)

\begin{tabular}{|c|c|c|c|}
\hline PHYLUM/ SUBPHYLUM/Clase/Orden & HA & RD & HS \\
\hline ESPONJAS (PORIFERA) & 58 & 99 & 107 \\
\hline CELENTERADOS (CNIDARIA) & 76 & 193 & 202 \\
\hline Hidrozoos (Hydrozoa) & 5 & 21 & 22 \\
\hline Escifozoos (Scyphozoa) & 2 & 5 & 6 \\
\hline Antozoos (Anthozoa) & 69 & 167 & 174 \\
\hline Estoloníferos (Stolonifera) & 3 & 5 & 7 \\
\hline Alcionáceos (Alcyonacea) & 1 & 6 & 6 \\
\hline Gorgonáceos (Gorgonacea) & 14 & 64 & 67 \\
\hline Zoantídeos (Zoanthidea) & 2 & 4 & 4 \\
\hline Anémonas (Actiniaria) & 8 & 12 & 12 \\
\hline Corales (Scleractinea) & 37 & 69 & 70 \\
\hline Coralimorfarios (Corallimorpharia) & 3 & 3 & 3 \\
\hline Anémonas de tubo (Ceriantharia) & 0 & 1 & 1 \\
\hline Corales negros (Anthipataria) & 1 & 3 & 4 \\
\hline CTENÓFOROS (CTENOPHORA) & 0 & 1 & 1 \\
\hline SIPUNCÚLIDOS (SIPUNCULA) & 0 & 2 & 2 \\
\hline MOLUSCOS (MOLLUSCA) & 142 & 514 & 566 \\
\hline Quitones (Polyplacophora) & 4 & 23 & 23 \\
\hline Gasterópodos (Gastropoda) & 123 & 337 & 384 \\
\hline Bivalvos (Bivalvia) & 10 & 140 & 143 \\
\hline Escafópodos (Scaphopoda) & 1 & 6 & 6 \\
\hline Cefalópodos (Cephalopoda) & 4 & 8 & 10 \\
\hline ANÉLIDOS (ANNELIDA) & 12 & 16 & 26 \\
\hline Poliquetos (Polychaeta) & 12 & 16 & 26 \\
\hline ARTRÓPODOS (ARTHROPODA) & 130 & 295 & 380 \\
\hline Picnogónidos (Pycnogonida) & 0 & 2 & 2 \\
\hline
\end{tabular}




\begin{tabular}{lrrr} 
CRUSTÁCEOS (CRUSTACEA) & 130 & 293 & 378 \\
Branquiópodos (Branchiopoda) & 2 & 1 & 2 \\
Ostrácodos (Ostracoda) & 0 & 1 & 1 \\
Copépodos (Copepoda) & 41 & 29 & 60 \\
Cirripedios (Cirripedia) & 2 & 6 & 6 \\
Estomatópodos (Stomatopoda) & 4 & 10 & 12 \\
Camarones (Stenopodidea y Caridea) & 19 & 56 & 66 \\
Langostas (Palinura, Astacidea & & & \\
y Thalassinidea) & 5 & 11 & 14 \\
Anomuros (Anomura) & 10 & 33 & 40 \\
Cangrejos (Brachyura) & 23 & 122 & 129 \\
Miscidáceos (Myscidacea) & 1 & 3 & 4 \\
Anfípodos (Amphipoda) & 1 & 0 & 1 \\
Isópodos (Isopoda) & 22 & 21 & 43 \\
UROCORDADOS (UROCHORDATA) & 8 & 8 & 13 \\
Tunicados (Ascidiacea) & 8 & 8 & 13 \\
QUETOGNATOS (CHAETOGNATHA) & 3 & 1 & 4 \\
EQUINODERMOS (ECHINODERMATA) & 73 & 124 & 154 \\
Crinoideos (Crinoidea) & 8 & 19 & 23 \\
Asteroideos (Asteroidea) & 17 & 22 & 33 \\
Ofiuroideos (Ophiuroidea) & 19 & 24 & 30 \\
Equinoideos (Echinoidea) & 21 & 42 & 51 \\
Holoturias (Holothuroidea) & 8 & 17 & 17 \\
BRAQUIÓPODOS (BRACHIOPODA) & 1 & 2 & 3 \\
PECES (PISCES) & 554 & 700 & 901 \\
Tiburones y rayas (Chondrichthyes) & 29 & 41 & 46 \\
Óseos (Osteichthyes) & 540 & 659 & 855 \\
\hline TOTAL ESPECIES & 1057 & 1955 & 2359 \\
Total taxa & 39 & 43 & 44 \\
\hline & & &
\end{tabular}


Sin embargo, a nivel de algunos grupos particulares de invertebrados sí existe un nivel de conocimiento bastante avanzado. Nuestros registros de 50 especies someras de gorgonáceos (de un total de 67), tienen una afinidad de hasta un $90 \%$ con las 42 especies reportadas para Jamaica (Kinzie, 1973), las 35 de Puerto Rico (González-Brito, 1970) y las 52 de Cuba (Alcolado, 1981). La lista de los corales de la Hispaniola con 70 especies está al nivel de las listas de Jamaica (Goreau y Wells, 1967) y Cuba (Zlatarski y Martínez-Estalella, 1982) y guarda entre un 70 a $80 \%$ de similitud con trece localidades caribeñas cuando se compara por métodos de clasificación numérica (Herrera, 2000). La fauna de poliplacóforos, representada por 23 especies, puede considerarse bien estudiada al compararla con Puerto Rico (Kaas, 1972) y Cuba (Espinosa et al., 1994), con quienes comparte una afinidad mayor del $80 \%$. También existe un conocimiento avanzado de los erizos, representados en nuestro trabajo por 51 especies (ver Herrera y Betancourt, 2004), respecto a las 69 reportadas para Cuba (MCTMA, 1998).

Los resultados de este proyecto constituyen un importante punto de partida para complementar y enriquecer los Informes y las Estrategias Nacionales de la Biodiversidad de República Dominicana (ONAPLAN, 1998) y Haití (Ministere de l'Environnement, 1998) a la luz de los planteamientos de la Convención de Diversidad Biológica (CBD, 2004).

El Proyecto HISPABIOTA continúa en su Fase II: Actualización de inventarios de grupos particulares, con el objetivo de perfeccionar y validar la información general de la Fase I y realizar un análisis más profundo y especializado de cada grupo taxonómico, obteniéndose inventarios actualizados que constituyen el producto terminado de nuestro proyecto y retroalimentan el inventario global. Al presente ya han sido publicadas las listas actualizadas, referenciadas y con localidades 
y mapas de distribución, de anémonas (Herrera y Betancourt, 2002), estomatópodos (Herrera y Betancourt, 2003) y equinodermos (Herrera y Betancourt, 2004) que junto al inventario de las algas bentónicas de la Hispaniola, previamente publicado (Betancourt y Herrera, 2001), irán formando una relación completa de la biota de la Isla.

Se puede obtener una idea de la importancia de este trabajo si consideramos que, incluyendo a las algas, HISPABIOTA ha inventariado por primera vez para la Hispaniola unas 2,766 especies pertenecientes a 49 grupos taxonómicos. Para República Dominicana, estas cifras incluyen 2,299 especies que prácticamente duplican el inventario de 1,200 especies de CIBIMA (1994) adicionando 1,068 nuevos registros, mientras que para Haití constituyen posiblemente el primer intento de recopilar su biota marina. Todos estos resultados constituyen una guía para proyectar nuevas investigaciones taxonómicas, ecológicas y zoogeográficas que den respuesta a los vacíos de información existentes y contribuyan -sobre bases científicasa la conservación, manejo y uso racional de la rica biodiversidad marina de la Isla Hispaniola.

\section{AGRADECIMIENTOS}

Nuestro agradecimiento a todas las personas e instituciones -nacionales e internacionales- que de una forma u otra contribuyeron durante estos tres años de trabajo a la obtención de datos de especies, literatura o nos orientaron en la búsqueda de información. Agradecemos la colaboración de investigadores y el personal de las bibliotecas del Grupo Jaragua, CEBSE, PRONATURA, INDOTEC, Centro de Investigaciones de Biología Marina (CIBIMA), Instituto de Oceanología de Cuba, Colegio de la Frontera Sur en México y las Universidades de Carolina del Este, Louisiana, Cornell y Copenhague. Agrade- 
cemos a los Departamentos de Botánica y Zoología de Invertebrados del Museo Nacional de Historia Natural del Smithsonian Institution. Reconocemos finalmente a la Sra. Lisette Betancourt y el Sr. Josué San Martín, sus gestiones bibliográficas en la Universidad de Miami.

\section{REFERENCIAS}

Alcolado, P. M. 1981. Zonación de los gorgonáceos someros de Cuba y su posible uso como indicadores comparativos de tensión hidrodinámica sobre los organismos del bentos. Inf. Cient. Téc., 187: $1-43$.

Betancourt, L. y A. Herrera 2001. Algas marinas bentónicas (Rhodophyta, Phaeophyta y Chlorophyta) conocidas para la Hispaniola. Revista Moscosoa, Jardín Botánico Nacional, 12: 105-134.

CBD 2004. Convention of Biological Diversity. United Nations Environmental Programme http://www.biodiv.org/

CIBIMA 1994. Estudio preliminar sobre la biodiversidad costera y marina de la República Dominicana. Editora Alfa y Omega, Santo Domingo, 459 pp.

Claro, R. 1994. Características generales de la ictiofauna. En: Ecología de los peces marinos de Cuba. Centro de Investigaciones de Quintana Roo, CIQRO, México, pp. 55-70.

Eschmeyer, W. N. (editor) 1998. Catalog of fishes. California Academy of Sciences, San Francisco, California, Vols. I, II y II, 2904 pp.

Espinosa, J., R. Fernández-Garcés y E. Rolán 1994. Catálogo de los moluscos marinos actuales de Cuba. Cuad. Invest. Biol. Bilbao, 18: 85-154.

González-Brito, P. 1970. Una lista de los octocorales de Puerto Rico. Carib. J. Sci. 10(1-2): 63-69.

Goreau, T. F. y J. W. Wells 1967. The shallow water scleractinea of Jamaica: revised list of species and their vertical distribution range. Bull. Mar. Sci., 17(2): 442-453. 
Herrera, A. 2000. Reclasificación de la biodiversidad coralina caribeña incluyendo los datos de la Hispaniola. En: La clasificación numérica y su aplicación en la ecología. Programa EcoMar, Inc./ Univ. INTEC, Impresora Sanmerycar, Santo Domingo, 88 pp.

Herrera, A. y L. Betancourt 2002. Especies de anémonas (Coelenterata: Actiniaria: Corallimorpharia, Zoanthidea y Ceriantharia) conocidas para la Hispaniola. Rev. Cien. Soc., Univ. INTEC, (27) 3: 439-452.

Herrera, A. y L. Betancourt 2003. Especies de estomatópodos (Crustacea: Malacostraca: Stomatopoda) conocidas para la Hispaniola. Rev. Cien. Soc.Univ. INTEC, 28(2): 271-278.

Herrera, A. y L. Betancourt 2004. Especies de equinodermos recientes (Echinodermata: Crinoidea: Asteroidea: Ophiuroidea: Echinoidea y Holothuroidea) conocidas para la Hispaniola Rev. Cien. Soc. Univ. INTEC, 29.

Kaas, P. 1972. Polyplacophora of the Caribbean region. Studies on the Fauna of Curacao and other Caribbean Islands, 137: 1-162.

Kinzie, R. 1973. The zonation of West Indian gorgonians. Bull. Mar. Sci., 23(1): 93-155.

MCTMA 1998. Informe Nacional a la Cuarta COP del Convenio Sobre Diversidad Biológica, Ministerio de Ciencia Tecnología y Medio Ambiente de Cuba, 16 pp.

Ministere de l'Environnement 1998. Implantation de la Convention sur Diversité Biologique en Haití. Rapport Intérimaire a la Quatrième Conférence des Parties. Republique d'Haïti, Ministere de L'Environnement, $5 \mathrm{pp}$.

ONAPLAN 1998. Primer Informe Nacional sobre la Implementación de la Convención de Diversidad Biológica en República Dominicana. Oficina Nacional de Planificación, Santo Domingo, 10 pp.

Ruppert, E. E. y R. D. Barnes 1994. Invertebrate Zoology. Sexta Edición, Saunders College Publishing, 1056 pp.

Zlatarski, V. N. y Martínez-Estalella, N. 1982. Les Scleractíniaires de Cuba. Edición de la Academia de Ciencias de Bulgaria, Sofía, 312 pp. 